http://jmscr.igmpublication.org/home/

ISSN (e)-2347-176x ISSN (p) 2455-0450

crossref DOI: https://dx.doi.org/10.18535/jmscr/v10i1.27

Journal Of Medical Science And Clinical Research

\title{
Perioperative Management of Pulmonary Embolism Following Bipolar Cemented Hemiarthroplasty- A Case Report
}

Authors

\author{
Dr Nisha Krishnaraj ${ }^{1}$, Dr Afreen Nahar $R^{2}$, Dr Arun Kumar ${ }^{3}$ \\ ${ }^{1}$ Post Graduate, ${ }^{2}$ Assistant Professor, ${ }^{3}$ Assosiate Professor \\ Department of Anaesthesia, Chettinad Hospital and Research Institute
}

\section{Introduction}

Perioperative pulmonary emboli (PE) in trauma patients are common. Pulmonary embolism is a potentially lethal complication and accounts for many cases of postoperative deaths each year. Pulmonary embolism (PE) describes an obstruction of the pulmonary arterial tree with abnormal material (thrombus, tumour, air or fat), usually originating somewhere else in the body. Patients can present acutely (immediately after the event), sub-acutely (within days/weeks after the embolism) or chronically (years after theembolism). The most common cause of acute $\mathrm{PE}$ is the migration of thrombus from veins (or right heart) to the pulmonary arteries. The risk factors for pulmonary embolism are immobility, malignancy, surgery, hospitalization, pregnancy, advancing age, trauma, infection, oestrogen, smoking, obesity, blood transfusion, hyper viscosity syndromes. The Virchow triad risk factors of pulmonary embolism are venous stasis, endothelial injury and hypercoagulable states. Patients with trauma, particularly to the long bones and pelvis, who are bed ridden and immobile for a prolonged period of time are at greatest risk of developing venous thromboembolism. The following case report describes the anaesthetic events and management of a patient posted for bipolar hemiarthroplasty who developed pulmonary embolism intra operatively immediately following cementing.

\section{Case Report}

83yrs old female who was a known case of hypertension and type II Diabetes Mellitus for past $15 y$ rs on regular medication was apparently normal 5 days prior to presenting to the hospital. She had a history of slip and fall at house and injury to left side neck of femur. Patient was admitted in hospital and all necessary investigations were done. She had a past history of lumbar vertebral fracture and stabilization done 10 years prior, of which no reports were available during current admission. She had a receding jaw with edentulous mouth and less pad of cheek fat. Her neck was short. All base line blood investigations were done and found to be normal. The patient gave history suggestive of METS less than 4. Patient's ECG showed T wave inversion in V3-V6 and lead III. Echocardiogram was done which showed Ejection fraction of $60 \%$ with mild MR and mild TR, Concentric LVH was present. Cardiologist opinion was obtained and was advised coronary angiogram in view of ECG changes. The patient refused to undergo coronary 
angiogram prior to fracture fixation. Hence, she was taken up for surgery under high cardiac risk. Considering the high cardiac risk and prior history of lumbar vertebral surgery, the choice of anesthesia for this surgery was general anesthesia with ultrasound guided suprainguinal facia iliaca block.

Patient was shifted to operation theatre. One large intravenous cannula was secured. All base line monitors were connected. Patient was premedicated with midazolam $1 \mathrm{mg}$, fentanyl $50 \mathrm{mcg}$. Radial artery cannulation was done after premedicating the patient. Invasive blood pressure monitoring was done throughout the surgery. Patient was induced with thiopentone $125 \mathrm{mg}$ and Isoflurane $2 \%$. Endotracheal tube was facilitated with succinyl choline $100 \mathrm{mg}$ intravenous. After administering general anaesthesia, under ultrasound guidance, supra inguinal fascia iliaca block was given with injection $0.25 \%$ ropivacaine $30 \mathrm{ml}$. Anaesthesia was maintained with oxygen, air, isoflurane and intermittent bolus of vecuronium. The patient's initial invasive blood pressure was $170 / 90 \mathrm{mmHg}$ she was given $10 \mathrm{mg}$ esmolol and 4mg Metaprolol after which blood pressure was maintained around 140/90mmHg thereafter. Two hours intra operatively during cementing patient developed sudden hypotension with IBP of $70 / 50 \mathrm{mmHg}$, tachycardia with heart rate of 120bpm and hypoxia with $94 \% \mathrm{SpO} 2$ with $100 \%$ oxygen. Injection phenylephrine 40 micrograms was given intravenously to treat hypotension. $300 \mathrm{ml}$ fluid bolus was given. Hydrocortisone $200 \mathrm{mg}$ IV was given immediately. Hypotension and hypoxia improved in $10 \mathrm{~min}$. There was persistent tachycardia throughout the surgery.ETCo2 was between 23-26 mmHg.ABG was taken intraoperatively and $\mathrm{PaCo} 2$ was found to be $55 \mathrm{mmHg}, \mathrm{Ph} 7.24$, pO2 $112 \mathrm{mmHg}$ with fiO2 60\%, HCO3 $21.2 \mathrm{mmol} / \mathrm{L}$. Paracetamol $1 \mathrm{~g}$ and fentanyl $40 \mathrm{mcg}$ were given IV intraoperatively for analgesia. At the end of the surgery, patient's vital signs were near normal, respiratory efforts were good, hence she was planned for ex-tubation. Patient was extubated after reversal with $0.5 \mathrm{mg}$ glycopylorate and $2.5 \mathrm{mg}$ neostigmine IV. Patient was stable after extubation. Patient was shifted to intensive care unit for further monitoring. Postoperatively after one hour patient developed sudden tachycardia, hypotension, breathlessness, she was drowsy but aurosable. Patient was started on noradrenaline starting from $0.02 \mathrm{mic} / \mathrm{kg} / \mathrm{min}$ and tritrated according to blood pressure. Echocardiogram was done and showed right atrium and right ventricle dilation with normally functioning left ventricle. 12 lead ECG was taken which also showed changes with sinus tachycardia S1Q3T3, RBBB, $\mathrm{T}$ wave inversion in lead II, III and Avf, ST depression in V2-V5.Doppler for lower limb was done and there was no evidence of deep vein thrombosis. Although no thrombus was visualized in the heart chambers on echocardiogram, taking into account the dilated right heart chambers, ECG showing right heart strain along with the clinical presentation of the patient was started on injection unfractionated heparin $4000 \mathrm{U} \quad(10 \mathrm{U} / \mathrm{kg})$ intravenously QID. She required noradrenaline support for 24 hours post-operatively. D-Dimer level was done and value was $>5000 \mathrm{U}$. Since DDimer levels can be inherently raised following surgery, CTPA was done the next day which showed thrombus in segmental and subsegmental branches of pulmonary artery in right lower lobe. After 4 days of receiving unfractionated heparin, patient was given injection enoxaparin $0.6 \mathrm{ml}$ BD subcutaneously for 5 days. She was transferred to the ward after 5 days. She recovered well thereafter and was discharged from the ward after 10 days on tablet Rivaroxaban $15 \mathrm{mg}$ BD for 21 days. Then patient was on tablet Rivaroxaban 20mg OD.

\section{Discussion}

Hip fractures are common problems that are already challenging the medical system as the population ages. In this case the patient already had vertebral fracture and stabilization was done so general anesthesia with nerve block for pain management was preferred for this patient. 
Diagnosing pulmonary embolism is clinically challenging during intra operative period and is more often missed, being an incidental autopsy finding. The survival rate of pulmonary embolism is very poor with three-month mortality being $17.1 \%$. The outcome depends upon degree of vascular obstruction and associated right ventricular strain. In severe pulmonary obstruction, the right ventricle needs to generate asystolic pressure over $50 \mathrm{mmHg}$ to maintain adequate cardiac output. About $85 \%$ of deaths due to massive pulmonary embolism occurs within the first 6 hours. The main goal of treatment in massive pulmonary embolism is to improve the forward flow and to reduce the after load in right ventricle, so as to avoid right ventricular failure.

Intraoperative features of pulmonary embolism are sudden unexplained hypotension, hypoxaemia, bronchospasm and a decrease in end tidal carbondioxide levels. Our patient was immobile for 5 days following fracture she was not started on any thrombolytic prophylaxis. This may be one of the reasons for intraoperative pulmonary embolism. The increased intramedullary pressure during cementing can also be a reason for emboli. In this case there is a significant decrease in ETCO2 with increase in $\mathrm{PaCo} 2$ this is called as "separation phenomenon" during general anesthesia, which means a decrease trend in ETCO2 and increase in $\mathrm{PaCO} 2$.

Since it was a hip surgery, the intra-operative pulmonary embolism might also be caused by cement embolus. The known bone cement implantation syndrome (BCIS) is commonly caused by PE, which is characterized by a number of clinical features such as hypoxia, hypotension, cardiac arrhythmias and increased pulmonary vascular resistance. It usually occurs at one of these stages of surgical procedure including femoral reaming, acetabular or femoral cement implantation, insertion of the prosthesis or joint reduction. However, in this case, the hemodynamic changes and hypoxia presented at the stage of reaming but patient recovered after half an hour and the condition further worsened after the patient is shifted to intensive care unit and immediate echocardiogram and electrocardiogram confirmed the diagnosis. CTPA gave the definitive diagnosis.

\section{Conclusion}

Immobilized patients with long bone fracture are the population at high risk for pulmonary emboli than other patients. The mortality following a pulmonary embolism depends on several factors, including the size of the embolus, the number of arteries blocked, and the overall health of the patient. Proper thrombolytic prophylaxis should be started prior to surgery. The current practice of withholding anticoagulation prior to surgery in trauma patients with orthopedic injuries at high risk of pulmonary embolism is not indicated. The risk/benefit ratio of preoperative anticoagulation must be carefully considered and weighed against the risk of pulmonary embolism.

\section{References}

1. Kakkar VV, Corrigan TP, Fossard DP Sutherland I. Prevention of fatal postoperative pulmonary embolism by low doses of heparin. Lancet1975;12(2): 5679.

2. Lassen M, Borris L, Nakov, R. Use of the low-molecular weight heparin reviparin to prevent deep-vein thrombosis after leg injury requiring immobilization. $\mathrm{N}$ Engl $\mathrm{J}$ of Med 2002;16(347):726-30.

3. Barash P, Cullen B, Stoelting R. Anaesthetic consideration in Pulmonary embolism. Cinical anesthesia. 4th ed. Philadelphia Lippincott, Williams, \& Wilkins; 2001. 450 - 69.

4. Stricker K, Korlen R, Yen K. Severe Hypercapnia Due to pulmonary embolism of Poly methylmethacrylate during vertebroplasty Anesth Analg 2004;7(98): 1184- 1186.

5. Samuel Z. Goldhaber, Elliott CG, Acute Pulmonary Embolism: Part I. 
Epidemiology, Pathophysiology, Diagnosis and Circulation 2003; 108:2726.

6. Gregoire LG, Perrier A. Contemporary approach to the diagnosis of non massive pulmonary embolism. Curr Opin Pulm Med 2006 (12)291-298.

7. Perruchoud C, Blanc.C, Transesophageal Echocardiography for the diagnosis and management of massive pulmonary embolism. Anesth Analg2006;8 (103): 3839.

8. Kearon C. Natural history of venous thromboembolism. Circulation 2003; 3(107):I-22-1-30.

9. Riber C, Alstrup N, Nymann T, Bogstad JW, Wille Jorgensen P, Tonnesen $\mathrm{H}$. Postoperative thromboembolism after day case herniorrhapy. Br J Surg.1996;6 (83): 420-421.

10. Konstantinides S, Meyer G, Becattini C, et al. 2019 ESC Guidelines for the diagnosis and management of acute pulmonary embolism developed in collaboration with the European Respiratory Society(ERS). Eur Heart J, 2019; 1 e61.

11. Putu Astawa. Bali Medical Journal (Bali Med J) 2020, Volume 9, Number 2: 520523P-ISSN.2089-1180, E-ISSN.23022914. 